\title{
Magnetic resonance imaging of brain angiogenesis after stroke
}

\author{
Peter R. Seevinck $\cdot$ Lisette H. Deddens • \\ Rick M. Dijkhuizen
}

Received: 27 April 2010/ Accepted: 1 June 2010/Published online: 16 June 2010

(C) The Author(s) 2010. This article is published with open access at Springerlink.com

\begin{abstract}
Stroke is a major cause of mortality and longterm disability worldwide. The initial changes in local perfusion and tissue status underlying loss of brain function are increasingly investigated with noninvasive imaging methods. In addition, there is a growing interest in imaging of processes that contribute to post-stroke recovery. In this review, we discuss the application of magnetic resonance imaging (MRI) to assess the formation of new vessels by angiogenesis, which is hypothesized to participate in brain plasticity and functional recovery after stroke. The excellent soft tissue contrast, high spatial and temporal resolution, and versatility render MRI particularly suitable to monitor the dynamic processes involved in vascular remodeling after stroke. Here we review recent advances in the field of MR imaging that are aimed at assessment of tissue perfusion and microvascular characteristics, including cerebral blood flow and volume, vascular density, size and integrity. The potential of MRI to noninvasively monitor the evolution of post-ischemic angiogenic processes is demonstrated from a variety of in vivo studies in experimental stroke models. Finally, we discuss some pitfalls and limitations that may critically affect the accuracy and interpretation of MRI-based measures of (neo)vascularization after stroke.
\end{abstract}

Keywords Angiogenesis - Magnetic resonance imaging Stroke - Brain ischemia - Microvessel density .

\footnotetext{
P. R. Seevinck $(\varangle) \cdot$ L. H. Deddens $\cdot$ R. M. Dijkhuizen $(\varangle)$ Biomedical MR Imaging and Spectroscopy Group, Image Sciences Institute, University Medical Center Utrecht, Yalelaan 2, 3584 CM Utrecht, The Netherlands e-mail: P.Seevinck@umcutrecht.nl

R. M. Dijkhuizen

e-mail: rick@invivonmr.uu.nl
}

\begin{abstract}
Microvessel size - Cerebral blood volume . Cerebral blood flow . Blood-brain barrier leakage . MRI
\end{abstract}

\section{Introduction}

Stroke is the result of occlusion or rupture of a brain artery, leading to loss of focal blood flow and brain function. Despite significant neurological deficits, most patients experience at least some degree of spontaneous recovery [1], which may be augmented by therapeutic intervention [2]. Currently, neuroprotective therapy after acute clinical stroke is restricted to the use of a single therapeutic agent, recombinant tissue plasminogen activator (rtPA), which should be given within $4.5 \mathrm{~h}$ after stroke to limit risk of hemorrhagic transformation [3]. However, experimental studies suggest that restorative pharmacological and cellbased therapies have potential to improve functional outcome when initiated $24 \mathrm{~h}$ up to weeks after stroke [2]. Such therapies amplify certain endogenous processes, some of which are also activated in the developing brain [4], that may contribute to spontaneous recovery after stroke. These events not only involve neuronal reorganization, but also include vascular remodeling through angiogenesis. Interestingly, there is an increasing amount of studies that has provided evidence for formation of new blood vessels after cerebral ischemia, and a possible significant role of angiogenesis in post-stroke recovery [5-10]. However, the exact pattern of neovascularization and its relation to restoration of function after stroke are still largely unresolved. Therefore, thorough characterization of the spatial and temporal profile of angiogenesis after ischemic stroke is of significant importance to elucidate its role in recovery and remodeling of neuronal tissue and ensuing functional 
outcome, which may lead to new insights for further development of neurorestorative therapies.

Magnetic resonance imaging (MRI) provides a powerful tool to noninvasively assess the evolution of various indices that can characterize cerebral tissue status after stroke [11-14]. Over the last decade, different MRI methods have also been applied to measure angiogenesis-related processes. This review describes results from such studies in experimental stroke models and discusses the principles, potentials and pitfalls of MRI methods to assess brain angiogenesis after stroke. First, the physiological process of angiogenesis after stroke will be briefly introduced. The main emphasis will be on the methodology and application of different MR imaging techniques, with and without contrast enhancement, that enable noninvasive monitoring of developing and mature microvasculature for stroke research.

\section{Angiogenesis after stroke}

Angiogenesis is the formation of new blood vessels from existing vessels, which is a normal and vital process in tissue growth and development that may also occur under pathophysiological conditions, such as after stroke. The physiological cascade of angiogenesis after stroke is highly complex and has already been extensively described in recent reviews [5, 6, 8-10]. Here we restrict the description of post-stroke angiogenesis to a brief introduction of the stages of onset, formation and maturation of new blood vessels (schematically illustrated in Fig. 1). We refer to the

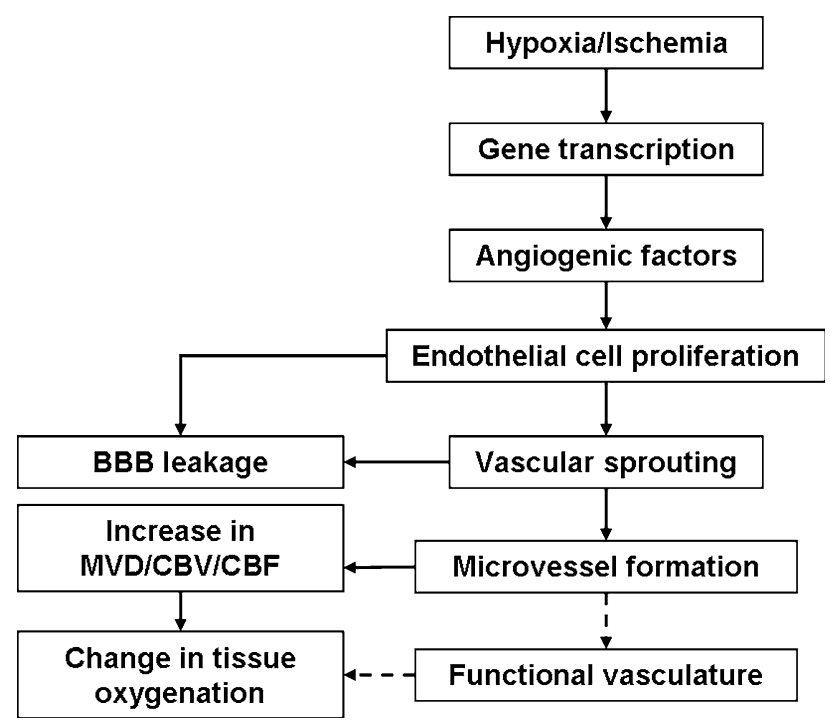

Fig. 1 Schematic representation of the cascade of events associated with angiogenesis after ischemic stroke. The physiological phenomena on the left side may be assessed with MRI ( $B B B$ blood-brain barrier, $M V D$ microvessel density, $C B V$ cerebral blood volume, $C B F$ cerebral blood flow) above reviews and references therein for more detailed explanation of the molecular and cellular aspects of angiogenesis after stroke.

Upon cessation of blood flow, the hypoxic/ischemic condition around a stroke lesion rapidly triggers transcription of a variety of genes that may be involved in the process of angiogenesis [6]. For example, the production of polypeptide growth factors, such as vascular endothelial growth factor (VEGF), and proinflammatory cytokines by residing brain cells and/or infiltrating inflammatory cells, creates a permissive environment for sprouting of proliferating endothelial cells $[6,8,10]$. It has been shown in rodent stroke models that proliferating endothelial cells form vascular buds that connect with small microvessels a few days after stroke (Fig. 2a-c). This stage of early angiogenesis is associated with a highly leaky blood-brain barrier (BBB) $[15,16]$. From 1 week after stroke, significant increases in microvessel density have been reported [8]. With time of survival, the conglomerates of microvessels increase in size (Fig. 2d), potentially giving rise to an increased cerebral blood volume (CBV) and flow (CBF) $[17,18]$. Furthermore, a decrease in vessel permeability can be seen over time [8], which is suggestive for remodeling of pericytes, astrocytes and other cells that are involved in BBB integrity.

Importantly, formation of new vessels after stroke may (1) contribute to recovery of tissue-at-risk by restoring metabolism in surviving neurons, (2) facilitate removal of necrotic debris, and/or (3) enhance supply of neurotrophic compounds for neuronal remodeling (e.g. synaptogenesis and neurite sprouting) $[5,6,8-10,19]$. However, whether angiogenesis indeed gives rise to full-fledged functional vascular networks around a stroke lesion is still unclear and remains an important topic for further research.

\section{MRI-based assessment of brain angiogenesis after stroke}

In the last two decades, MRI has proven to be a valuable tool to investigate the spatiotemporal profile of ischemiainduced changes after stroke, mainly attributable to its capability to longitudinally evaluate a wide spectrum of structural and functional tissue characteristics. This versatility originates from the fact that contrast in MR images is dependent on intrinsic, biophysical tissue properties such as proton density, inter- and intramolecular magnetic interactions, oxygenation state, magnetic susceptibility, diffusion, perfusion and flow. These endogenous tissue characteristics influence the MRI signal by their effect on MR relaxation times $\left(T_{1}, T_{2}\right.$ and $\left.T_{2}^{*}\right)$ and water proton mobility, which can be exploited to generate image contrast. Additionally, exogenous contrast agents (e.g. gadolinium chelates and 
Fig. 2 Scanning electron micrographs of vascular casts of rat brains after unilateral occlusion of the middle cerebral artery (MCA). Three days after MCA occlusion, vascular budding was visible at many sites in the ipsilateral cortex, involving both small and large vessels (a white arrows).

Microvessels formed connections with surrounding proliferating vessels (b, c white arrows). With time of survival, the conglomerates of microvessels increase in size, forming a dense and chaotic microvasculature surrounding larger microvessels $(\mathbf{d})$. Inserted bars denote the magnification in each figure. Reproduced from Ref. [77] with permission from Lippincott, Williams and Wilkins
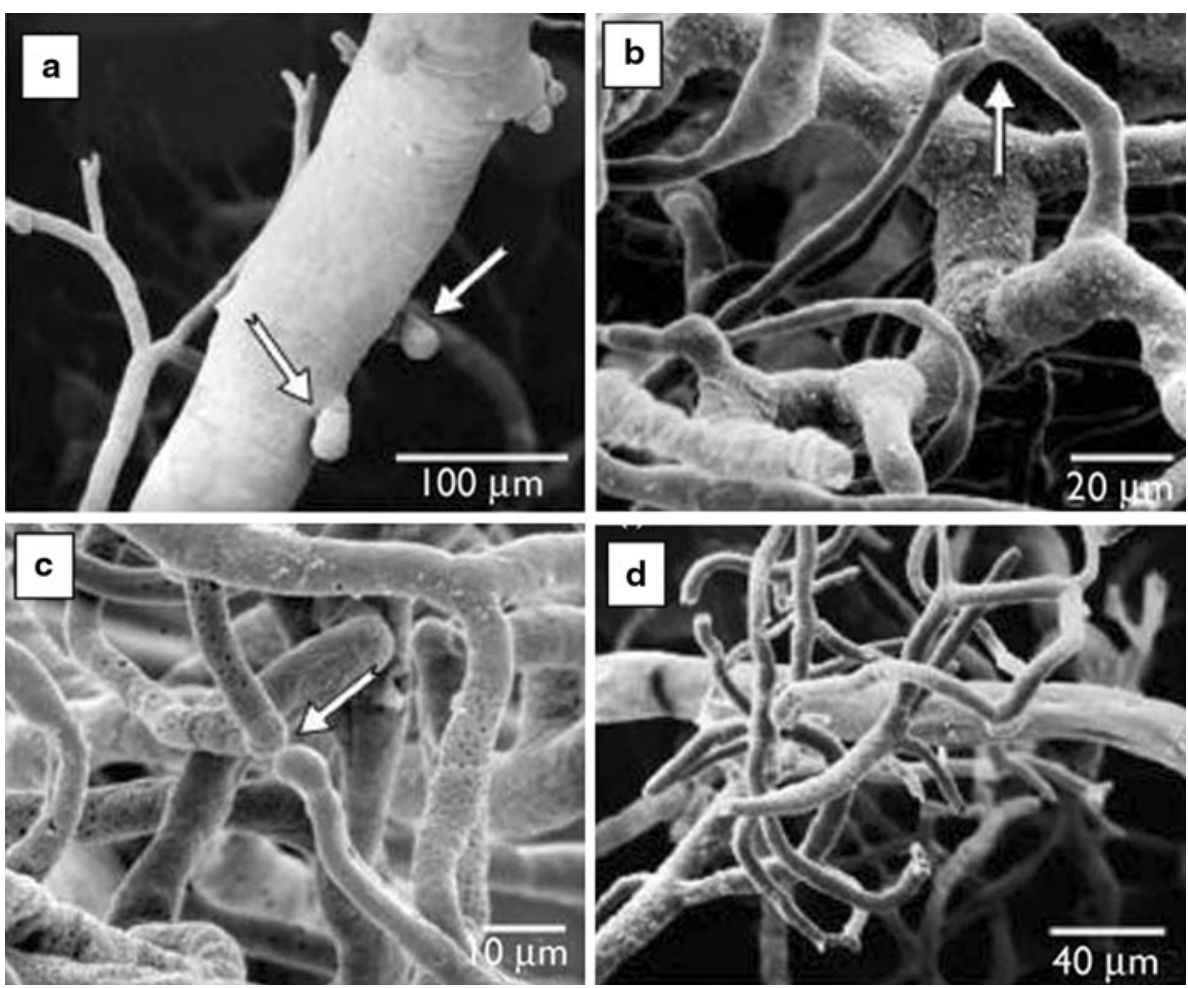

iron oxide particles) can be applied to enhance endogenous contrast-generating mechanisms. This can improve anatomical and/or physiological distinction, and may allow detection of otherwise indiscernible factors. In stroke research, both endogenous and exogenous MR contrast mechanisms have been exploited to assess cerebrovascular changes associated with angiogenesis. In the following sections the various MR imaging techniques that have been utilized to assess angiogenesis in the brain will be reviewed.

\section{MRI of vessels, blood volume and perfusion}

Probably the most intuitive way to evaluate the presence of angiogenic vessels is direct visualization of the newly formed vasculature by MR angiography [20]. However, the spatial resolution of MRI is currently limited to approximately $50 \mu \mathrm{m}$ for small animal MR imaging, and to approximately $250 \mu \mathrm{m}$ on clinical MRI scanners. Since angiogenic processes in brain tissue, such as endothelial sprouting and microvessel formation, take place at length scales that are at least one order of magnitude lower, direct depiction of remodeling of cerebrovascular structures is currently not feasible with MRI. Alternatively, hemodynamic parameters that are directly affected by angiogenesis, such as cerebral blood volume (CBV) and cerebral blood flow $(\mathrm{CBF})$, can be measured with perfusion MR imaging techniques such as dynamic susceptibility contrastenhanced (DSC-)MRI, steady state susceptibility contrast- enhanced (ssCE-)MRI and arterial spin labeling (ASL). DSC- and ssCE-MRI are based on detection of signal changes induced by intravascularly injected exogenous contrast agents, while ASL exploits endogeneous contrast mechanisms involving magnetically labeled arterial water. The following paragraphs provide a brief introduction to these MRI methods. For more detailed information on the principles of the different perfusion MRI techniques, we refer the reader to specific reviews [21-23].

Dynamic susceptibility contrast-enhanced MRI

DSC-MRI allows calculation of cerebral hemodynamic parameters from the time-course of signal changes induced by the first passage of a paramagnetic contrast agent after intravenous injection [24]. The most commonly applied exogenous contrast agents are gadolinium chelates [25]. Their high magnetic susceptibility induces local magnetic field inhomogeneities. The consequent local shortening of $T_{2}$ and $T_{2}^{*}$ leads to enhanced MR signal decay. By dynamically monitoring these signal changes in perfused tissue during the first passage of a bolus of injected contrast agent, hemodynamic parameters such as $\mathrm{CBF}, \mathrm{CBV}$ and mean transit time (MTT) can be estimated from the linear relationship between contrast agent concentration and change in effective transverse relaxation rate $\left(\Delta R_{2}^{*}=\Delta\left(1 / T_{2}^{*}\right)\right.$, and the central volume theory [24, 25].

DSC-MRI has been successfully applied for spatiotemporal monitoring of changes in $\mathrm{CBV}$ in relation to 
Fig. 3 Longitudinal changes in vascular density and $\mathrm{CBF}$ after 60 min of transient MCA occlusion in rats. Excised rat brains clearly show enhanced vascular density on the cortical surface of the ipsilateral hemisphere after MCA occlusion (a). Relative CBF maps (b) calculated from ASL experiments demonstrate a significantly increased CBF in the ipsilateral cortex from day 1 up to day 14 after MCA occlusion (c). Reproduced from Ref. [18] with permission from Lippincott Williams and Wilkins
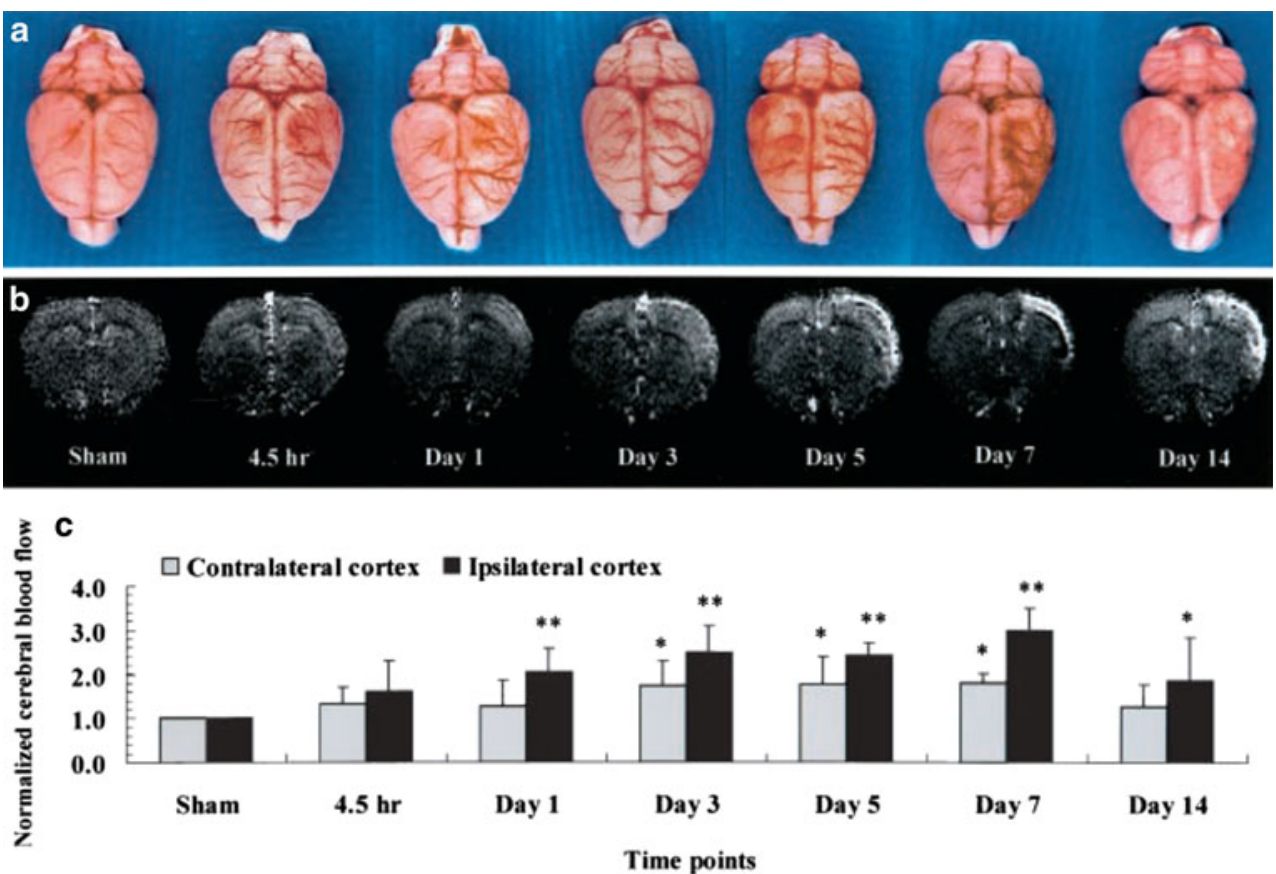

formation of new blood vessels in brain tumors, where a significant correlation was found between increase of relative CBV and histologically determined microvessel density and fractional blood volume [26]. In a rat stroke model, Lin et al. demonstrated with DSC-MRI that CBV was significantly enhanced in the ipsilateral cerebral cortex at 7 days after transient unilateral occlusion of the middle cerebral artery (MCA), which correlated with increased vessel density in the outer cortical layer [18]. Similarly, DSC-MRI has been applied to measure changes in CBV in a rat embolic stroke model after angiogenesis-promoting treatment with neuronal progenitor cells [27]. A coincident increase of CBV and vascular density was reported, suggesting a relationship between the elevated CBV and angiogenesis [27].

\section{Arterial spin labeling}

As mentioned above, $\mathrm{CBF}$ can also be determined with MRI without the use of exogenous contrast agents. In ASL, radiofrequency $(\mathrm{RF})$ pulses are used to magnetically alter the status of water protons in arterial blood with respect to those in stationary tissue water, thereby generating an endogenous intravascular tracer [28, 29]. Assuming that the magnetically labeled arterial blood exchanges with tissue water at the level of the capillaries, CBF can in principle be quantified from a labeling experiment and a control experiment without magnetically labeled water protons, based on the theory of diffusible tracer kinetics [28, 30, 31].

ASL has been applied in a number of experimental stroke studies to estimate $\mathrm{CBF}$ in relation to angiogenesis.
In conjunction with an increased vascular density, which is clearly visible on the surface of the ipsilateral brain surface in Fig. 3a, Lin et al. demonstrated a significantly enhanced $\mathrm{CBF}$ in the perilesional cortex from day 1 to 14 after transient MCA occlusion in rats (Fig. 3b, c) [18]. Chopp and co-workers have reported that treatment with the vasodilatator sildenafil enhanced angiogenesis and selectively increased the CBF level in the ischemic boundary in rats after embolic stroke [17, 32]. Furthermore, co-localization of the area with CBF improvement with regions with increased fractional anisotropy of white matter tissue was reported, pointing towards a link between angiogenesis and neuronal reorganization $[32,33]$.

Despite the significant information on tissue perfusion, MRI-based measurement of hemodynamics provides relatively low specificity for angiogenesis. For example, CBV and $\mathrm{CBF}$ increases may also arise in response to autoregulatory vasodilation or vessel recruitment due to arteriogenesis. Therefore, alternative MR methodologies that allow assessment of structural features of the microvasculature that may be more specifically related to angiogenesis have been recently proposed, which will be the subject of the following paragraph.

\section{Steady state susceptibility contrast-enhanced MRI}

As opposed to measurement of tissue perfusion from the dynamics of MR signal decay due to first passage of a contrast agent in DSC-MRI, ssCE-MRI allows estimation of blood volume, microvessel density and vessel size from steady state contrast-induced signal changes. In ssCE-MRI, 
transverse relaxation rates $R_{2}$ and $R_{2}^{*}$ are measured before and after administration of an intravascular contrast agent that establishes a high susceptibility difference, for quantitative purposes [34], and has a long blood half-life, for a prolonged acquisition time-window [35-37]. To that aim, ultrasmall superparamagnetic iron oxide (USPIO) particles have been most frequently applied [23]. The long circulating USPIOs generate a static magnetic field inhomogeneity around the vessels. Such field inhomogeneities affect spin echo and gradient echo transverse relaxation rates $\left(R_{2}\right.$ and $R_{2}^{*}$, respectively) in a different way, dependent on many factors including concentration and susceptibility of the contrast agent, vessel diameter, fractional blood volume, tissue diffusion coefficient, magnetic field strength and MR imaging protocol (see references [38-41] for detailed MR physical background information on intravascular susceptibility contrast mechanisms). With mathematical modeling and Monte Carlo simulations it has been shown that changes in the transverse relaxation rate $R_{2}$ (i.e. $\Delta R_{2}$ ), as a result of the application of an intravascular susceptibility contrast agent, are predominantly sensitive to small vessels $(<10 \mu \mathrm{m})$, while changes in the transverse relaxation rate $R_{2}^{*}$ (i.e. $\Delta R_{2}^{*}$ ) are sensitive to vessels of all sizes [34, 38-42]. This sensitivity of $\Delta R_{2}$ for small vessels was exploited by Dunn et al. to enable quantification of the CBV increase associated with hypoxia-induced angiogenesis in rat brain, based on measurements of $\Delta R_{2}$ in brain parenchyma and serum, and a verified linear dependence between $\Delta R_{2}$ and contrast agent concentration [43]. When the local $\Delta R_{2}^{*}$ and susceptibility difference $(\Delta \chi)$ are known, it has been shown from theory and simulations that the total blood volume fraction (BVf) can be estimated from the following equation [40, 41, 44].

$\mathrm{BVf}=3 / 4(\pi) \Delta R_{2}^{*} /\left(\gamma \Delta \chi B_{0}\right)$

In rat brain tumors, blood volume fractions determined using Eq. 1 were shown to correlate reasonably well with histological measurements [45, 46].

Beside estimation of blood volume, ssCE-MRI can provide specific details on vascular morphology. Dennie et al. have demonstrated that the ratio of the change in $R_{2}^{*}$ and $R_{2}$ before and after contrast agent injection, i.e. $\Delta R_{2}^{*} / \Delta R_{2}$, directly relates to microvessel morphology [47], as had originally been predicted based on mathematical modeling [38]. A significant correlation between an increase of the $\Delta R_{2}^{*} / \Delta R_{2}$ ratio and an increase of the average histological vessel diameter was observed inside tumor tissue [47], which was attributed to enlarged diameters of angiogenic vessels [44-47]. Tropres et al. further developed this concept of vessel size imaging based on steady state contrastenhanced relaxation rate shifts [44, 48], and defined the vessel size index (VSI), a measure of the average vessel radius [48].
$\operatorname{VSI}(\mu \mathrm{m})=0.425\left(D / \gamma \Delta \chi B_{0}\right)^{1 / 2}\left(\Delta R_{2}^{*} / \Delta R_{2}\right)^{3 / 2}$

Although good correlations between VSI and the histological vessel size have been reported [44], calculations of VSI have been based on estimations of the intravascular susceptibility difference $(\Delta \chi)$ before and after injection of contrast agent, and the local diffusion coefficient $(D)[34,48,49]$. Since $\Delta \chi$ is difficult to determine under most in vivo conditions, Jensen and Chandra introduced the ratio $Q \equiv \Delta R_{2} /\left(\Delta R_{2}^{*}\right)^{2 / 3}$, which for a sufficiently high $\Delta \chi$, only depends on intrinsic tissue properties, and which should correlate with microvessel density (MVD) [49].

$\operatorname{MVD}\left(\mathrm{mm}^{-2}\right) \approx Q^{3} /(4.725 D)$

Wu et al. have assessed the MVD in normal mouse brain based on the ratio $Q$, using a literature value for $D=0.664 \mu \mathrm{m}^{2} / \mathrm{ms}$ [50]. This provided an average brain MVD of $282 \pm 43 / \mathrm{mm}^{2}$, which is in reasonable agreement with histologically determined values.

Recently, two studies have employed ssCE-MRI to assess vascular remodeling after experimental stroke [51, 52]. In a serial study in rats recovering from transient cerebral ischemia performed by Lin et al., an initial decrease in vascular density (based on the $Q$ value) and an increase in vessel size (based on VSI) was observed in the reperfused cortex at day 1 and 3 [52]. Immunohistological analysis confirmed a similar decrease in microvessel density and increase in size of vessels with a diameter larger than $30 \mu \mathrm{m}$. These observations were explained by a more pronounced effect of edema on compression of small capillaries as compared to large-sized vessels, leading to a shift in the calculated average vessel size. A significant increase of total CBV from day 3 to 14 in the affected hemisphere, based on $\Delta \mathrm{R}_{2}^{*}$, was speculated to be caused by improvement of collateral circulation in the relatively large microvessels. At days 14 and 21 , increases in $\Delta \mathrm{R}_{2}$ (microvascular $\mathrm{CBV}$ ) and $Q$ (microvascular density) were noticed in the reperfused cortex, which was attributed to the surge of angiogenesis. In contrast, Bosomtwi et al. observed a lowered $Q$-based and histology-based MVD in recovered ischemic tissue at 2 weeks after embolic stroke in rats [51]. A possible explanation is that only a small part of the recovered region was highly angiogenic, which may have been obscured by the analysis of a relatively large region-of-interest [51].

The potential of ssCE-MRI to monitor changes in CBV, microvessel density and vessel size in a single experiment, is demonstrated in Fig. 4. We performed ssCE-MRI in rats at 7 days after $60 \mathrm{~min}$ transient unilateral MCA occlusion. $R_{2}$ and $R_{2}^{*}$ maps were acquired pre- and post-administration of $16.5 \mathrm{mg} / \mathrm{kg}$ USPIO (Guerbet, Aulnay-sous-Bois, France). Figure 4 shows exemplary data from a rat with a 

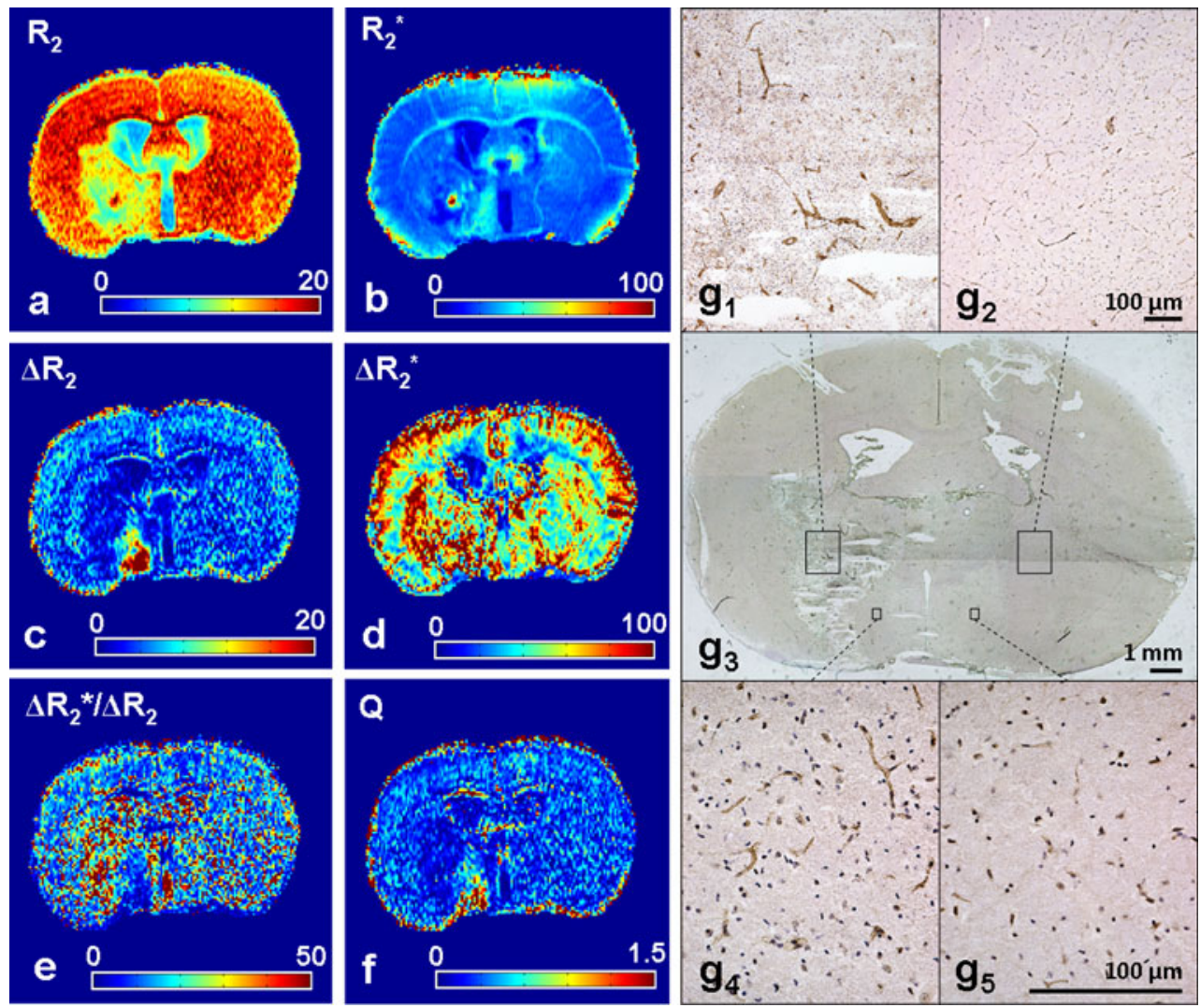

Fig. 4 MRI and histology of a coronal rat brain slice at 7 days after 60min unilateral MCA occlusion, resulting in a subcortical infarct. Precontrast $R_{2}$ map displays the subcortical lesion with decreased $R_{2}(\mathbf{a})$. The hyperintense spot inside the lesion on the pre-contrast $R_{2}$ and $R_{2}^{*}$ maps reflects a bleeding (a and $\mathbf{b}$ ). The enhanced $R_{2}^{*}$ in the ipsilateral hypothalamus may be a sign of increased venous blood volume (b). This area exhibited an increased $\Delta R_{2}$ value after administration of a superparamagnetic blood pool agent (USPIO), pointing toward an increase in the density of microvessels with a relatively small diameter (c). The high contrast-induced $\Delta R_{2}^{*}$ in the entire lesion is reflective of

subcortical infarct. The pre-contrast $R_{2}$ map clearly depicts a unilateral lesion in the caudate putamen, characterized by a decreased $R_{2}$ value, i.e. prolonged $T_{2}$, associated with vasogenic edema [13] (Fig. 4a). A hyperintense spot in the center of the lesion, which also revealed a high $R_{2}^{*}$ value on the pre-contrast $R_{2}^{*}$ map (Fig. $4 b$ ), corresponded with a bleeding identified with histology (Fig. 4g). The increased $R_{2}^{(*)}$ can be explained by accumulation of paramagnetic deoxygenated blood [53]. The $\Delta R_{2}^{*}$ value (Fig. $4 \mathrm{~d}$ ), directly related to total BVf (Eq. 1), was increased in the lesion as compared to the contralateral hemisphere, suggestive of hyperperfusion. The ipsilateral hypothalamic region displayed an elevated $\Delta R_{2}$ (Fig. $4 \mathrm{c}$ ), which also revealed a high $Q$ value (Fig. 4f), suggestive of an enhanced microvascular $\mathrm{CBV}$ and microvessel density, respectively. These findings are in agreement with results presented by Lin increased total blood volume (d). The high $\Delta R_{2}^{*} / \Delta R_{2}$ ratio in the lesion is indicative of a relatively large vessel diameter (e), as was confirmed by histology with vessel staining with von Willebrand Factor (DABenhanced; brown) (nuclei were stained with hematoxylin (blue)) $\left(\mathbf{g}_{\mathbf{1}}\right)$. The ipsilateral hypothalamic region exhibited a low value on the $\Delta R_{2}^{*} /$ $\Delta R_{2}$ map, indicating a low vessel diameter. A high $Q$ value was observed in this area (f), indicative of an enhanced MVD, which was confirmed by histology $\left(\mathbf{g}_{4}\right)$. Contralaterally, normal vessel size $\left(\mathbf{g}_{2}\right)$ and density $\left(\mathbf{g}_{5}\right)$ were observed $\left(R_{2}, R_{2}^{*}, \Delta R_{2}\right.$ and $\Delta R_{2}^{*}$ are in units $\mathrm{sec}^{-1}$, $Q$ is in units $\sec ^{-1 / 3}$ )

et al. [52] as discussed in the previous paragraph. The relatively high pre-contrast $R_{2}^{*}$ value in this region may have been caused by an enhanced venous blood volume, as was suggested by Ding et al. [33], which will be further elaborated on in the next section. The $\Delta R_{2}^{*} / \Delta R_{2}$ map shows a low value in this area, pointing toward a low average vessel size (Fig. 4e). In contrast, a high $\Delta R_{2}^{*} / \Delta R_{2}$ value was observed in the remaining part of the lesion, which suggests that the average vessel size was relatively high. Histology confirmed substantial presence of larger vessels in the lesion (Fig. $4 \mathrm{~g}_{1}$ ), as well as an increased microvessel density (Fig. $4 \mathrm{~g}_{4}$ ) in the area with a high $Q$ value. Contralaterally, normal vessel size (Fig. $4 \mathrm{~g}_{2}$ ) and densities (Fig. $4 \mathrm{~g}_{5}$ ) were observed. Perls' Prussian Blue staining confirmed that USPIO had not extravasated (data not shown). 


\section{MRI of blood-brain barrier permeability and tissue oxygenation}

Next to assessment of hemodynamic and morphologic changes related to vascular remodeling, MRI can provide information on BBB permeability and oxygenation state of brain tissue, which both are involved in the process of angiogenesis. BBB permeability can be investigated by dynamic contrast-enhanced MRI (DCE-MRI), in which changes in MR signal, as a result of leakage of an intravascularly injected contrast agent into the interstitial space, are dynamically monitored [54]. The oxygenation state of brain tissue can be investigated by exploiting the high magnetic susceptibility of deoxygenated hemoglobin [55]. Since venous blood is rich of deoxygenated hemoglobin, blood oxygenation level-dependent (BOLD) MRI may detect newly developed vasculature that is rich of venous blood [33]. Both methods will be briefly introduced in relation to their potential to evaluate angiogenesis after stroke.

\section{Dynamic contrast-enhanced MRI}

DCE-MRI measures the time-course of contrast-induced changes of the $T_{1}$ relaxation time constant due to contrast agent extravasation into tissue. Since the tissue $T_{1}$ is directly related to the intravascular and parenchymal concentration of the contrast agent, one can estimate the bloodto-brain transfer constant, $K_{i}$, by locally assessing the dynamics of the signal intensity of a time-series of $T_{1}$ weighted MR images, or $T_{1}$ maps, with tracer kinetic models [54, 56, 57]. In experimental stroke research, DCEMRI with gadolinium chelates has been employed to quantify BBB integrity [16, 17, 27, 33, 52, 58-61]. BBB breakdown develops within hours after the onset of brain ischemia [16, 58, 62], which has been associated with a variety of pathophysiological processes, including the initial inflammatory response and upregulation of the vascular permeability factor VEGF [10, 63]. Interestingly, a significant increase of $K_{i}$ has been observed with DCE-MRI at later time points after stroke as well, which has been hypothesized to be associated with angiogenesis [17, 27, $33,52,59]$. Chopp and co-workers have reported that $K_{i}$ peaks around 2 weeks after experimental stroke, coincident with increases in $\mathrm{CBF}, \mathrm{CBV}$ and vessel density in the same regions, and they have proposed $K_{i}$ as an additional marker for angiogenesis [27, 59]. Experimental angiogenesis-promoting treatment with sildenafil was reported to accelerate the dynamics of the BBB leakage [33] (Fig. 5).

\section{Blood oxygenation level-dependent MRI}

With BOLD MRI, the local magnetic field disturbances induced by the relatively high magnetic susceptibility of deoxygenated hemoglobin are exploited, which provides $T_{2}$ contrast on spin echo images [64] and $T_{2}^{*}$ contrast on gradient echo images [55]. The effect observed on gradient echo images is much larger, since apart from dephasing

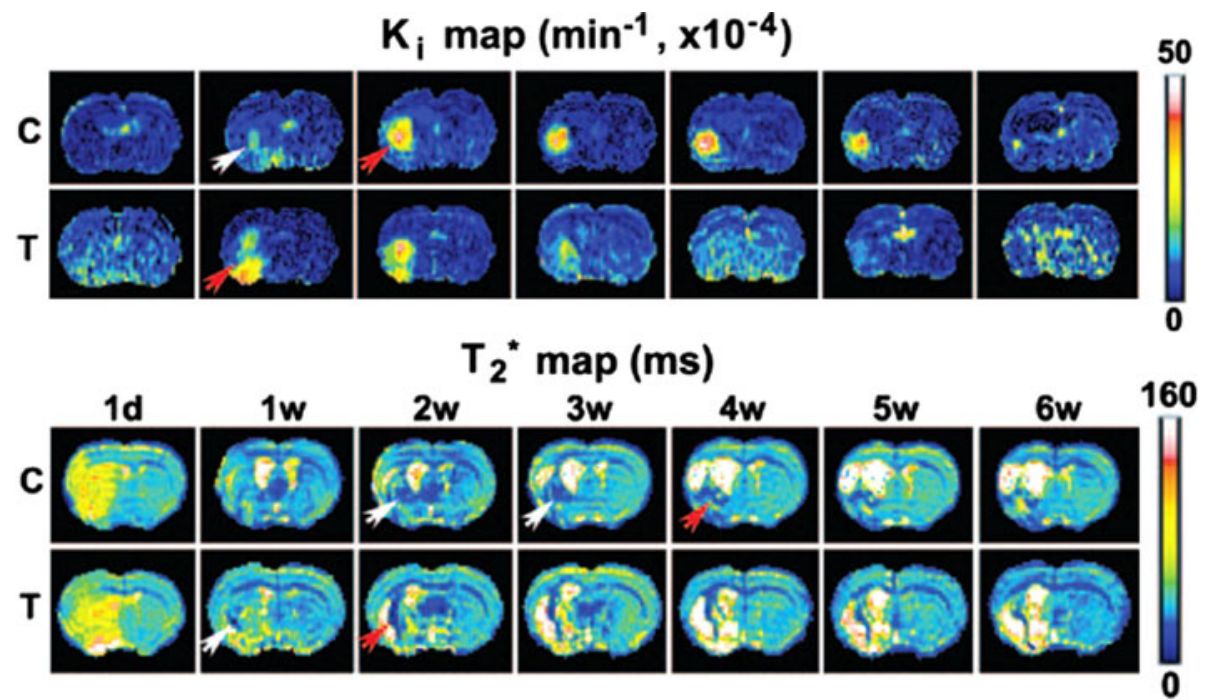

Fig. 5 Maps of $K_{i}$ and $T_{2}^{*}$ at different time points (1 day-6 weeks) after unilateral embolic stroke in a saline-treated control rat (C) and in a sildenafil-treated rat $(T)$. In the control rat (first row), $K_{i}$ inside the lesion was elevated from 1 week on, indicative of BBB disruption, which peaked between 2 and 5 weeks after stroke. In a rat treated with angiogenesis-promoting sildenafil (second row), increase of $K_{i}$ was observed earlier and lasted shorter. Red arrows indicate significantly increased $K_{i}$ values. In these presumably angiogenic regions, $T_{2}^{*}$ values were significantly decreased, at 4 or 2 weeks after stroke in saline- (third row) and sildenafil-treated rats (fourth row), respectively. Red arrows indicate significantly decreased $T_{2}^{*}$ values. Reproduced from Ref. [33] with permission from Lippincott Williams and Wilkins 
due to diffusion through local magnetic field gradients, all static dephasing is refocused in spin echo images [40, 64]. Ding et al. have recently evaluated the potential of $T_{2^{-}}^{*}$ weighted imaging to identify angiogenesis in post-stroke rat brain [33]. They observed a shorter $T_{2}^{*}$, indicative of more deoxygenated blood, in perilesional areas of placeboand sildenafil-treated rats starting at 1 and 2 weeks after stroke, respectively, which was hypothesized to arise from maturing angiogenic venous structures. This was verified with susceptibility weighted imaging, a variant of $T_{2^{-}}^{*}$ weighted MRI that includes phase information [65, 66], which clearly enhanced the contrast of the areas with increased magnetic susceptibility [33]. Furthermore, regions exhibiting a decreased $T_{2}^{*}$ value spatially matched with regions with high vascular permeability, based on $K_{i}$ measurements (Fig. 5). In another study from the same group, $T_{2}^{*}$ shortening in the ischemic hemisphere corresponded spatiotemporally with increasing $\mathrm{CBF}$ and tissue fractional anisotropy, which would reflect a close relationship between angiogenesis and neuronal remodeling [32] (Table 1).

\section{Discussion and future perspectives}

In the search for neuroprotective and neurorestorative therapies aiming to improve functional outcome after stroke, a better understanding of the endogenous processes involved in spontaneous recovery after stroke is of major importance. A healthy tissue perfusion status, responsible for providing nutrients and oxygen and allowing clean-up of waste products, is critical for mediating functional recovery after brain injury. This process could be significantly augmented by vascular remodeling through angiogenesis. Therefore, the prospect to noninvasively assess physiological and structural characteristics of such events would improve our knowledge on brain plasticity, and may eventually help us to direct functional outcome of stroke to our benefit. This review illustrates how MRI can be applied to noninvasively monitor the evolution of various postischemic processes related to angiogenesis in the brain. The presented studies demonstrate that MR imaging enables assessment of a variety of (micro)vascular characteristics, such as cerebral blood flow and volume, vascular density and vessel size, BBB integrity and blood oxygenation.

Despite its potential, we need to also be aware of several pitfalls and limitations related to the application of MRI as a tool for evaluating angiogenesis after stroke. For example, an increase in $K_{i}$, i.e. contrast agent leakage, and shortening of $T_{2}^{*}$, i.e. more deoxygenated blood, which has been found in relation to angiogenesis [33], may also arise as a result of $\mathrm{BBB}$ disruption and hemorrhage in response to vascular pathology [67]. Furthermore, MRI measurements of tissue perfusion and microvascular characteristics (CBF, CBV, $K_{i}, \mathrm{MVD}$ and VSI) rely on algorithms that are based on relatively basic biophysical and mathematical models that may be inaccurate under complex or altered conditions, as is the case in stroke pathophysiology. The methodological difficulties related to the models used in DSC-MRI and ASL to quantify perfusion, especially under abnormal flow conditions, have been extensively reviewed [21, 22, 68-70]. Furthermore, from a physiological point of view, an increase in $\mathrm{CBF}$ and $\mathrm{CBV}$, which has been associated with post-stroke angiogenesis, may not be exclusively specific to neovascularization, as vasodilatation of existing vessels and collateral flow may also contribute. Hence, the combination of perfusion imaging with additional MR methods that provide information on microvessel density and vessel size may help elucidating the complex interdependence between $\mathrm{CBF}, \mathrm{CBV}, \mathrm{MVD}$ and

Table 1 MRI techniques, parameters, and contrast types and mechanisms to assess tissue characteristics related to vascular remodeling through angiogenesis

\begin{tabular}{|c|c|c|c|c|c|}
\hline \multirow[t]{2}{*}{ MRI technique } & \multicolumn{2}{|l|}{ Parameter } & \multicolumn{2}{|l|}{ Contrast } & \multirow[t]{2}{*}{ References } \\
\hline & Physiological & MRI & Mechanism & Type & \\
\hline Angiography & Vascular anatomy & - & Tracer inflow & $\begin{array}{c}\text { Endogenous/ } \\
\text { exogenous }\end{array}$ & {$[20]$} \\
\hline DSC-MRI & $\begin{array}{l}\mathrm{CBF} \\
\mathrm{CBV}\end{array}$ & $\Delta R_{2}^{*}$ & $\Delta \chi$, contrast passage & Exogenous & {$[17,18,32,52,78]$} \\
\hline ASL & $\mathrm{CBF}$ & $\begin{array}{l}\text { Magnetically labeled } \\
\text { arterial water }\end{array}$ & Tracer inflow & Endogenous & {$[18,32,33,78]$} \\
\hline ssCE-MRI & $\begin{array}{l}B V f_{\text {total }} \\
B V f_{\text {microvascular }} \\
\text { Vessel size } \\
\text { Vessel density }\end{array}$ & $\begin{array}{l}\Delta R_{2}^{*} \\
\Delta R_{2} \\
\Delta R_{2}^{*} / \Delta R_{2} \\
\Delta R_{2} /\left(\Delta R_{2}^{*}\right)^{2 / 3}\end{array}$ & $\Delta \chi$, pre- and post-contrast & Exogenous & $\begin{array}{l}{[45,46]} \\
{[43]} \\
{[34,44-48]} \\
{[49-52]}\end{array}$ \\
\hline DCE-MRI & BBB permeability & $K_{i}$ & $T_{1}$, pre- and post-contrast & Exogenous & {$[16,17,27,33,52,54,57-61]$} \\
\hline BOLD & Oxygenation & $\Delta R_{2}, \Delta R_{2}^{*}$ & $\Delta \chi$, deoxyHb & Endogenous & {$[33,55,64,79]$} \\
\hline
\end{tabular}


VSI. This can be accomplished with ssCE-MRI, as discussed in this review. Theoretical modeling as well as Monte Carlo simulations have demonstrated the ability to quantify MVD and VSI with ssCE-MRI [34, 42, 44, 48, 49], however, in practice MVD and VSI are typically qualitatively expressed [47, 52]. Quantification of microvessel density and size based on in vivo MRI measurements have been reported based on assumptions of the diffusion coefficient $\mathrm{D}$ and susceptibility difference $\Delta \chi[50$, 51]. Since $D$ may considerably change under specific pathological conditions, and post-contrast $\Delta \chi$ will vary between subjects, it can be expected that defining $D$ and $\Delta \chi$ based on literature values will affect the accuracy of quantitative MVD and VSI measurements. In addition, large variations of $Q$ values, as well as $\Delta R_{2}^{*} / \Delta R_{2}$ ratios, have been reported for rat brain [44, 48-52], which may be explained by additional biophysical assumptions and generalizations. First, the vascular network is assumed to consist of randomly oriented straight cylinders, ignoring the influence of curvature. Second, the vessel diameter within tissue-of-interest is often approximated at a single diameter. Third, isotropic diffusion is assumed. Fourth, computations rely on an intact BBB. Obviously, variations and alterations in these factors can lead to under- or overestimation of MVD and VSI calculations. Elucidation of the influence of these aspects in ssCE-MRI is critical for adequate use of this promising approach in assessing vascular characteristics in healthy and pathological brain.

A promising new MRI approach that may enable direct detection of angiogenesis is molecular imaging with contrast agents that are targeted to specific molecular markers of vascular remodeling. In oncology, MR-based molecular imaging with $\alpha_{v} \beta_{3}$-integrin-targeted paramagnetic nanoparticles has already demonstrated its potential to monitor tumor angiogenesis [71-73]. A clear example of the potential of in vivo molecular imaging of angiogenesis after stroke has recently been presented by Cai et al., who used positron emission tomography (PET) to measure upregulation of VEGF receptors with radioactively-labeled VEGF in the ischemic rat brain. For noninvasive monitoring of kinetics of expression of molecular markers, nuclear imaging techniques are valuable, however, their relatively low resolution, requirement of a radioactive tracer and lack of anatomical reference, are significant drawbacks that may be overcome by development of MR-based molecular imaging. A potential innovative way to monitor the onset of vascular remodeling in a very early stage with MRI, may be accomplished with MR reporter genes, which allow noninvasive assessment of expression of a gene of interest together with synthesis of an MR detectable by-product, such as endogenous paramagnetic ferritin [74-76].

In conclusion, in this review we have presented recent developments in the field of MR imaging that enable in vivo assessment of different physiological parameters, e.g. tissue perfusion and microvascular morphology, that can shed light on various aspects related to angiogenesis after stroke. These developments may provide important new insights into the role of vascular remodeling in brain plasticity and functional recovery after stroke, and may be valuable for monitoring of possible future therapies designed to promote (neo)vascularization in stroke patients.

Acknowledgments The research leading to these results has received funding from the Netherlands Organization for Scientific Research (NWO) (VIDI 917.76.347), the Netherlands Ministries of Economic Affairs and Education, Culture and Science through the NL Agency project TARGET MR-IT, and the European Union's Seventh Framework Programme (FP7/2007-2013) under grant agreements no 201024 and no 202213 (European Stroke Network). Annette van der Toorn, Ivo Tiebosch, Bas Oude Munnink and Elga de Vries are gratefully acknowledged for technical support and advice, as well as Laboratoire Guerbet (Aulnay-sous-Bois, France) for kindly providing the USPIO contrast agent P904.

Open Access This article is distributed under the terms of the Creative Commons Attribution Noncommercial License which permits any noncommercial use, distribution, and reproduction in any medium, provided the original author(s) and source are credited.

\section{References}

1. Cramer SC (2008) Repairing the human brain after stroke: I. Mechanisms of spontaneous recovery. Ann Neurol 63:272-287

2. Cramer SC (2008) Repairing the human brain after stroke. II. Restorative therapies. Ann Neurol 63:549-560

3. Hacke W, Kaste M, Bluhmki E et al (2008) Thrombolysis with alteplase 3 to 4.5 hours after acute ischemic stroke. N Engl J Med 359:1317-1329

4. Cramer SC, Chopp M (2000) Recovery recapitulates ontogeny. Trends Neurosci 23:265-271

5. Arai K, Jin G, Navaratna D et al (2009) Brain angiogenesis in developmental and pathological processes: neurovascular injury and angiogenic recovery after stroke. FEBS J 276:4644-4652

6. Beck H, Plate KH (2009) Angiogenesis after cerebral ischemia. Acta Neuropathol 117:481-496

7. Krupinski J, Kaluza J, Kumar P et al (1994) Role of angiogenesis in patients with cerebral ischemic stroke. Stroke 25:1794-1798

8. Slevin M, Kumar P, Gaffney J et al (2006) Can angiogenesis be exploited to improve stroke outcome? Mechanisms and therapeutic potential. Clin Sci (Lond) 111:171-183

9. Hayashi T, Deguchi K, Nagotani S et al (2006) Cerebral ischemia and angiogenesis. Curr Neurovasc Res 3:119-129

10. Zhang Z, Chopp M (2002) Vascular endothelial growth factor and angiopoietins in focal cerebral ischemia. Trends Cardiovasc Med 12:62-66

11. Bardutzky J, Shen Q, Henninger N et al (2007) Characterizing tissue fate after transient cerebral ischemia of varying duration using quantitative diffusion and perfusion imaging. Stroke 38:1336-1344

12. Farr TD, Wegener S (2010) Use of magnetic resonance imaging to predict outcome after stroke: a review of experimental and clinical evidence. J Cereb Blood Flow Metab 30(4):703-717 
13. Dijkhuizen RM, Nicolay K (2003) Magnetic resonance imaging in experimental models of brain disorders. J Cereb Blood Flow Metab 23:1383-1402

14. Baird AE, Warach S (1998) Magnetic resonance imaging of acute stroke. J Cereb Blood Flow Metab 18:583-609

15. Sandoval KE, Witt KA (2008) Blood-brain barrier tight junction permeability and ischemic stroke. Neurobiol Dis 32:200-219

16. Durukan A, Marinkovic I, Strbian D et al (2009) Post-ischemic blood-brain barrier leakage in rats: one-week follow-up by MRI. Brain Res 1280:158-165

17. Li L, Jiang Q, Zhang L et al (2007) Angiogenesis and improved cerebral blood flow in the ischemic boundary area detected by MRI after administration of sildenafil to rats with embolic stroke. Brain Res 1132:185-192

18. Lin TN, Sun SW, Cheung WM et al (2002) Dynamic changes in cerebral blood flow and angiogenesis after transient focal cerebral ischemia in rats. Evaluation with serial magnetic resonance imaging. Stroke 33:2985-2991

19. Manoonkitiwongsa PS, Jackson-Friedman C, McMillan PJ et al (2001) Angiogenesis after stroke is correlated with increased numbers of macrophages: the clean-up hypothesis. J Cereb Blood Flow Metab 21:1223-1231

20. Oostendorp M, Post MJ, Backes WH (2009) Vessel growth and function: depiction with contrast-enhanced MR imaging. Radiology 251:317-335

21. Barbier EL, Lamalle L, Decorps M (2001) Methodology of brain perfusion imaging. J Magn Reson Imaging 13:496-520

22. Calamante F, Thomas DL, Pell GS et al (1999) Measuring cerebral blood flow using magnetic resonance imaging techniques. J Cereb Blood Flow Metab 19:701-735

23. Wu EX, Tang H, Jensen JH (2004) Applications of ultrasmall superparamagnetic iron oxide contrast agents in the MR study of animal models. NMR Biomed 17:478-483

24. Rosen BR, Belliveau JW, Vevea JM et al (1990) Perfusion imaging with NMR contrast agents. Magn Reson Med 14: 249-265

25. Villringer A, Rosen BR, Belliveau JW et al (1988) Dynamic imaging with lanthanide chelates in normal brain: contrast due to magnetic susceptibility effects. Magn Reson Med 6:164-174

26. Pathak AP, Schmainda KM, Ward BD et al (2001) MR-derived cerebral blood volume maps: issues regarding histological validation and assessment of tumor angiogenesis. Magn Reson Med 46:735-747

27. Jiang Q, Zhang ZG, Ding GL et al (2005) Investigation of neural progenitor cell induced angiogenesis after embolic stroke in rat using MRI. Neuroimage 28:698-707

28. Detre JA, Leigh JS, Williams DS et al (1992) Perfusion imaging. Magn Reson Med 23:37-45

29. Williams DS, Detre JA, Leigh JS et al (1992) Magnetic resonance imaging of perfusion using spin inversion of arterial water. Proc Natl Acad Sci USA 89:212-216

30. Calamante F, Williams SR, van Bruggen N et al (1996) A model for quantification of perfusion in pulsed labelling techniques. NMR Biomed 9:79-83

31. Buxton RB, Frank LR, Wong EC et al (1998) A general kinetic model for quantitative perfusion imaging with arterial spin labeling. Magn Reson Med 40:383-396

32. Ding G, Jiang Q, Li L et al (2008) Magnetic resonance imaging investigation of axonal remodeling and angiogenesis after embolic stroke in sildenafil-treated rats. J Cereb Blood Flow Metab 28:1440-1448

33. Ding G, Jiang Q, Li L et al (2008) Angiogenesis detected after embolic stroke in rat brain using magnetic resonance $\mathrm{T} 2 * \mathrm{WI}$. Stroke 39:1563-1568

34. Kiselev VG, Strecker R, Ziyeh S et al (2005) Vessel size imaging in humans. Magn Reson Med 53:553-563
35. Enochs WS, Harsh G, Hochberg F et al (1999) Improved delineation of human brain tumors on MR images using a long-circulating, superparamagnetic iron oxide agent. J Magn Reson Imaging 9:228-232

36. Corot C, Violas X, Robert P et al (2003) Comparison of different types of blood pool agents (P792, MS325, USPIO) in a rabbit MR angiography-like protocol. Invest Radiol 38:311-319

37. Hamberg LM, Boccalini P, Stranjalis G et al (1996) Continuous assessment of relative cerebral blood volume in transient ischemia using steady state susceptibility-contrast MRI. Magn Reson Med 35:168-173

38. Boxerman JL, Hamberg LM, Rosen BR et al (1995) MR contrast due to intravascular magnetic susceptibility perturbations. Magn Reson Med 34:555-566

39. Kennan RP, Zhong J, Gore JC (1994) Intravascular susceptibility contrast mechanisms in tissues. Magn Reson Med 31:9-21

40. Kiselev VG, Posse S (1999) Analytical model of susceptibilityinduced MR signal dephasing: effect of diffusion in a microvascular network. Magn Reson Med 41:499-509

41. Yablonskiy DA, Haacke EM (1994) Theory of NMR signal behavior in magnetically inhomogeneous tissues: the static dephasing regime. Magn Reson Med 32:749-763

42. Pathak AP, Ward BD, Schmainda KM (2008) A novel technique for modeling susceptibility-based contrast mechanisms for arbitrary microvascular geometries: the finite perturber method. Neuroimage 40:1130-1143

43. Dunn JF, Roche MA, Springett R et al (2004) Monitoring angiogenesis in brain using steady-state quantification of DeltaR2 with MION infusion. Magn Reson Med 51:55-61

44. Tropres I, Grimault S, Vaeth A et al (2001) Vessel size imaging. Magn Reson Med 45:397-408

45. Valable S, Lemasson B, Farion R et al (2008) Assessment of blood volume, vessel size, and the expression of angiogenic factors in two rat glioma models: a longitudinal in vivo and ex vivo study. NMR Biomed 21:1043-1056

46. Beaumont M, Lemasson B, Farion R et al (2009) Characterization of tumor angiogenesis in rat brain using iron-based vessel size index MRI in combination with gadolinium-based dynamic contrast-enhanced MRI. J Cereb Blood Flow Metab 29:1714-1726

47. Dennie J, Mandeville JB, Boxerman JL et al (1998) NMR imaging of changes in vascular morphology due to tumor angiogenesis. Magn Reson Med 40:793-799

48. Tropres I, Lamalle L, Peoc'h M et al (2004) In vivo assessment of tumoral angiogenesis. Magn Reson Med 51:533-541

49. Jensen JH, Chandra R (2000) MR imaging of microvasculature. Magn Reson Med 44:224-230

50. Wu EX, Tang H, Jensen JH (2004) High-resolution MR imaging of mouse brain microvasculature using the relaxation rate shift index Q. NMR Biomed 17:507-512

51. Bosomtwi A, Jiang Q, Ding GL et al (2008) Quantitative evaluation of microvascular density after stroke in rats using MRI. J Cereb Blood Flow Metab 28:1978-1987

52. Lin CY, Chang C, Cheung WM et al (2008) Dynamic changes in vascular permeability, cerebral blood volume, vascular density, and size after transient focal cerebral ischemia in rats: evaluation with contrast-enhanced magnetic resonance imaging. J Cereb Blood Flow Metab 28:1491-1501

53. Atlas SW, Thulborn KR (1998) MR detection of hyperacute parenchymal hemorrhage of the brain. AJNR Am J Neuroradiol 19:1471-1477

54. Tofts PS, Kermode AG (1991) Measurement of the blood-brain barrier permeability and leakage space using dynamic MR imaging. 1. Fundamental concepts. Magn Reson Med 17:357-367

55. Ogawa S, Lee TM, Kay AR et al (1990) Brain magnetic resonance imaging with contrast dependent on blood oxygenation. Proc Natl Acad Sci USA 87:9868-9872 
56. Patlak CS, Blasberg RG, Fenstermacher JD (1983) Graphical evaluation of blood-to-brain transfer constants from multipletime uptake data. J Cereb Blood Flow Metab 3:1-7

57. Tofts PS, Brix G, Buckley DL et al (1999) Estimating kinetic parameters from dynamic contrast-enhanced T(1)-weighted MRI of a diffusable tracer: standardized quantities and symbols. J Magn Reson Imaging 10:223-232

58. Kastrup A, Engelhorn T, Beaulieu C et al (1999) Dynamics of cerebral injury, perfusion, and blood-brain barrier changes after temporary and permanent middle cerebral artery occlusion in the rat. J Neurol Sci 166:91-99

59. Li L, Jiang Q, Zhang L et al (2006) Ischemic cerebral tissue response to subventricular zone cell transplantation measured by iterative self-organizing data analysis technique algorithm. J Cereb Blood Flow Metab 26:1366-1377

60. Ewing JR, Knight RA, Nagaraja TN et al (2003) Patlak plots of Gd-DTPA MRI data yield blood-brain transfer constants concordant with those of 14C-sucrose in areas of blood-brain opening. Magn Reson Med 50:283-292

61. Jiang Q, Ewing JR, Ding GL et al (2005) Quantitative evaluation of BBB permeability after embolic stroke in rat using MRI. J Cereb Blood Flow Metab 25:583-592

62. Belayev L, Busto R, Zhao W et al (1996) Quantitative evaluation of blood-brain barrier permeability following middle cerebral artery occlusion in rats. Brain Res 739:88-96

63. Dvorak HF, Brown LF, Detmar M et al (1995) Vascular permeability factor/vascular endothelial growth factor, microvascular hyperpermeability, and angiogenesis. Am J Pathol 146:1029-1039

64. Bandettini PA, Wong EC, Jesmanowicz A et al (1994) Spinecho and gradient-echo epi of human brain activation using bold contrast - a comparative-study at 1.5 T. NMR Biomed 7: $12-20$

65. Haacke EM, Xu Y, Cheng YC et al (2004) Susceptibility weighted imaging (SWI). Magn Reson Med 52:612-618

66. Reichenbach JR, Venkatesan R, Schillinger DJ et al (1997) Small vessels in the human brain: MR venography with deoxyhemoglobin as an intrinsic contrast agent. Radiology 204:272-277

67. Lee JM, Zhai G, Liu Q et al (2007) Vascular permeability precedes spontaneous intracerebral hemorrhage in stroke-prone spontaneously hypertensive rats. Stroke 38:3289-3291
68. Ostergaard L, Weisskoff RM, Chesler DA et al (1996) High resolution measurement of cerebral blood flow using intravascular tracer bolus passages. Part I: Mathematical approach and statistical analysis. Magn Reson Med 36:715-725

69. Ostergaard L, Sorensen AG, Kwong KK et al (1996) High resolution measurement of cerebral blood flow using intravascular tracer bolus passages. Part II: Experimental comparison and preliminary results. Magn Reson Med 36:726-736

70. Wu O, Ostergaard L, Koroshetz WJ et al (2003) Effects of tracer arrival time on flow estimates in MR perfusion-weighted imaging. Magn Reson Med 50:856-864

71. Mulder WJ, Strijkers GJ, Habets JW et al (2005) MR molecular imaging and fluorescence microscopy for identification of activated tumor endothelium using a bimodal lipidic nanoparticle. FASEB J 19:2008-2010

72. Sipkins DA, Cheresh DA, Kazemi MR et al (1998) Detection of tumor angiogenesis in vivo by alphaVbeta3-targeted magnetic resonance imaging. Nat Med 4:623-626

73. Winter PM, Schmieder AH, Caruthers SD et al (2008) Minute dosages of alpha(nu)beta3-targeted fumagillin nanoparticles impair Vx-2 tumor angiogenesis and development in rabbits. FASEB J 22:2758-2767

74. Gilad AA, Winnard PT Jr, van Zijl PC et al (2007) Developing MR reporter genes: promises and pitfalls. NMR Biomed 20:275-290

75. Cohen B, Dafni H, Meir G et al (2005) Ferritin as an endogenous MRI reporter for noninvasive imaging of gene expression in C6 glioma tumors. Neoplasia 7:109-117

76. Genove G, DeMarco U, Xu H et al (2005) A new transgene reporter for in vivo magnetic resonance imaging. Nat Med $11: 450-454$

77. Krupinski J, Stroemer P, Slevin M et al (2003) Three-dimensional structure and survival of newly formed blood vessels after focal cerebral ischemia. Neuroreport 14:1171-1176

78. Bratane BT, Walvick RP, Corot $\mathrm{C}$ et al (2010) Characterization of gadolinium-based dynamic susceptibility contrast perfusion measurements in permanent and transient MCAO models with volumetric based validation by CASL. J Cereb Blood Flow Metab 30:336-342

79. Prinster A, Pierpaoli C, Turner R et al (1997) Simultaneous measurement of DeltaR2 and DeltaR2* in cat brain during hypoxia and hypercapnia. Neuroimage 6:191-200 